\title{
Ueber das Verhalten des Epithels bei der Heilung von Linear- und Lanzenmesserwunden in der Hornhaut.
}

Von

E. Neese in Kiew.

Bierza Tafel $1-V$.

Die feineren Vorgänge bei der Heilung von Wunden in der Hornhaut, die dieser dureh ein Staarmesser beigebracht werden, sind zuerst bekanntlich durch Guterbock*) und durch Gussenbauer**) an Kaninchen und an Fröschen studirt worden. Die Frage nach dem Verhalten des Epithèls bei diesem Vorgange ist jedoch von diesen beiden Forschern in nicht genügendem Maase berücksichtigt, zum mindesten in nicht befriedigender und auch nicht übereinstimmender Weise gelöst worden. Güterbock, der das Vorhandensein einer "Epitheleinsenkung" zuerst constatirte und besonders betonte, führte das Zustandekommen derselben auf einen durchaus passiven Vorgang zurück und suchte diesen auf eine rein mechanische Weise zu erklären. Es sollte nämalich die Epitheleinsenkung einfach durch das Hineindrücken und Hineinzerren mit der Spitze des Messers entstanden sein. Doch schieu diese Erklärungsweise für unsere gebräuch-

*) Virchow's Areh., Bd. L.

*) Arch. f. klin. Chirurgie, Bd. XII. p. 791.

จ. Graefe's Archiv für OphthaImologie, XXXIII. 1. 
lichen Staarextractionswunden, wo nach geschenem Finstiche mit darauffolgender Contrapunction die Schnittfübrung von innen, von der Descemetis aus, nach aussen zu, gegen das Hornhautepithel hin, vor sich geht, schon a priori wenig für sich zu haben, während das thatsäehliche Vorbandensein einer derartigen Epitheleinsenkung auch an solchen Wunden durch 0 . Becker*) in seinem Atlas dargelegt wird. Es könnte, wie derselbe **) hervorhebt, die Güterbock'sche Erklärungsweise allenfalls eben nur für die Einstichstelle selbst gelten.

Einen gewissen Grad von Wahrscheinlichkeit bätte diese Darlegung vielleicht noch fur die Lanzenmesserwunden, wo ein solches Hineinpressen und -zerren des mehrschichtigen Oberflächenepithels mit dem keilförmig zugespitzten Instrumente in das Hornhautparenchym hinein wohl noch eher denkbar wäre. Bringt man aber in der That einem Kaninchen mit dem Lanzenmesser eine Paracenthesenwunde in der Hornhant bei, so findet sich nach 30 Stunden ein Epithelzapfen, oder -pfropf von einer Soliditat und Mächtigkeit (Fig. 6), die es schon ron Hause aus als sehr wenig wahrscheinlich erscheinen lassen, als handle es sich hier um einen rein mechanischen und bloss passiven Vorgang.

Untersucht man hingegen eine solche Wunde in der ersten Stunde nach geschehener Operation, und wemn dieselbe noch klafft, unter dem Mikroskope, so findet man dass das Epithel, ohne im geringsten in die Wunde „hineingezerrt" oder -gepresst zu sein, scharf an dem klaffenden Rande derselben absehneidet.

*) Atlas der topographischen Anatomie des Auges. Lief. 1. Taf, $\mathrm{V}$ und VI.

**) Graefe-Saemisch, Handbuch der Augenheilkunde, Bd. V, p. 389 . 
Es ist diese Thatsache für die nichtpenetrirenden Wunden der Hornhaut zuerst durch H. v. Wyss in Zürich*) nachgewiesen worden, wobei der betreffende Autor jedoch die Art seiner Schnitt- und Messerführung unerwähnt gelassen hat.

Gussenbauer, welcher der "Epithel-Einsenkung" eine grössere Bedentung überhaupt nicht zu vindiciren scheint und derselben erst gelegentlich der mit Gu terbock entstandenen Controverse ausführlicher gedenkt, erklärt dieselbe nur zum Theil als Oberflächenepithel, - und zwar als Einrollung desselben in die Wunde hinein, infolge einer Zurückziehung der durch den Schnitt durchtrennten Elastica anterior, und durch den zwischen Epithel und Cornealsubstanz bestehenden Elasticitätsunterschied bedingt. Zum anderen Theil jedoch ist nach ihm das, was Güterbock auch noch für "Epitheleinsenkung” hält, bloss scheinbar, und dadurch entstanden, dass eingewanderte Zellen epitheliale Formen angenommen haben.

Gégen den ersten Theil der Gussenbauer'schen Auseinandersetzung lässt sich allerdings wohl kaum etwas einwenden - and je schräger die Schnittführung, je länger die Wunde und je mehr sich dieselbe der Lappenform nähert, nm so mehr wird in der That eine bogenförmige Einsenkung oder gar eine Einrollung der oberflächlichen Schichten der überhängenden Wundlefze mitsammt dem Epithele stattfinden. Eine besondere Widerlegung aber des zweiten Theiles dieser Auffassung unterlasse ich an dieser Stelle, weil dieselbe sich aus der weiteren Darstellung von selbst ergeben soll.

Erwähnt soll nur noch werden, dass die gegenseitige Verschiebung der Wundränder auch eine gewisse Art von hakenförmiger Epitheleinsenkung bedingen kann,

*) Virchow's Archiv, Bd. LXIX. 
sowie dass bis zu einem gewissen Grade eine solche dadurch zu Stande kommen kann, dass nach jeder Seite von dem Wundcanale hin eine allmählich sich steigernde A ufquellung der Cornealsubstanz von statten geht, wie Becker*) seinerzeit bereits hervorgehoben hat.

Alle diese Darstellungs- und Erklärungsweisen lassen jedoch eine active Betheiligung des Epitheles an dem Wundheilungsprocesse ausser Betracht; hierin stimnen auch Güterbock und Gussenbauer vollkommen miteinander uberein. Und doch wird eine solche Betheiligung durch das von Becker in der ersten lieferung seines Atlas abgebildete und erläuterte Iwanow'sche Präparat einer Staarextractionswunde in hohem Grade wahrscheinlich gemacht.

Am besten aber überzengt man sich von der Thatsüchlichkeit dieser Voraussetzung durch das Thierexperiment und an der Hand einer vervollkommneten Methode.

Die Bildung des Epithelverschlusses.

Legt man einem Kaninchen mit dem Linearmesser, durch Einstich und Contrapunction, central oder peripher, eine perforirende Wunde in der Hornhaut an und verfolgt dieselbe von Stunde zu Stunde, so beobachtet man an derselben Folgendes:

In der ersten Stunde nach vollbrachter Operation schneidet das Epithel scharf an den Rändern der klaffenden Wunde ab.

In der vierten Stunde hat das Epithel in seiner Gesammtdicke die beiderseitigen Wundränder eben umgriffen.

*) Graefe-Sämisch, Handbuch der Augenheilkunde, Bd. V, p. 391 . 
In der sechsten bis zehnten Stunde steigt dasselbe in mehrfacher Sehicht successive an den Wandungen des Wundcanals beiderseits hinab, und in der zwölften Stunde hat es die Mitte der Gesammtlänge dieses letzteren fast erreicht. (Fig. 1.)

In der fünfzehnten Stunde ist diese Mitte des sich allmählich verengernden Wundcanales bereits überschritten und findet an dieser engsten Stelle desselben zuerst eine Ueberbrückung und darauf eine Auspolsterung mit zwei nebeneinander liegenden Epithelwülsten (d) statt. (Fig. 2.)

In den nun folgenden 24 Stunden erfolgt von dieser Stelle, vom Fundus des Wundeanales aus, eine Bewegung der Epithelien von unten nach oben, eine Emporhebung der geschilderten Wülste, (die übrigens ein constantes Vorkommniss nicht bilden), bis nach ungefähr 48 Stunden die ganze obere Halfte der Wunde ausgefüllt und das Gesammtniveau der Cornea annähernd erreicht worden ist, falls nämlich der Klaffungsgrad der Wunde eine gewisse Grenze nicht überschreitet.

Ein solcher zweitägiger Epithelverschluss ist durch จ. Wyss*), der uberhaupt zuerst die proliferative Betheiligung des Epitheles an dem Wundverschlusse zu beweisen suchte, in seiner Figur 3 ähnlich bereits dargestellt.

Vollkommen analog ist der Vorgang bei der Lanzenmesserwundè: denn wăhrend, wie schon oben angedeutet, das Epithel der erst vor einer Stunde angelegten Wunde am Rande dieser letzteren scharf abschneidet, hat es denselben in der vierten Stunde bereits umfasst (Fig. 4), um dann von Stunde zu Stunde tiefer hinabzusteigen, die Wandungen des Wundeanales trichterartig überziehend, bis dieser denn nach 24 Stunden nach unten zu an seiner

*) a. a. 0 . 
engsten Stelle verschlossen ist. Es bleibt jetzt oben in dem Trichter nur noch ein kleines Lumen ubrig, dass sich auch allmählich schliesst, bis ungefăhr nach 30 Stunden der solide Wundverschluss in Gestalt des oben bereits angedenteten epithelialen Zapfens hergestellt ist. (Eig. 6.)

Ist ein Hinabsteigen des Epithels sowohl in die Linear- als auch in die Lanzenmesserwunde einmal erwiesen, so taucht die Frage auf, wie tief, bis wie weit in die Wunde hinein dieses Hinabsteigen erfolgt und wodurch demselben eine Grenze gesetzt wird?

Das Epithel steigt in die Wunde so tief hinab, als bis es auf einen natürlichen mechanischen Widerstand stösst. Es wird dieser Widerstand durch folgende Um* stände und Verhältnisse gesetzt.

Durchschneidet man eine Cornea, gleichviel mit welchem Messer, ob nun mit dem linear- oder dem lanzenfôrmigen, ob nun in einer gegen die Corneallamellen senkrechten oder schrägen Ebene - jedoch stets in dieser einen Ebene verbleibend - so ergiebt sich, dass in Folge ungleichartiger Elastieitätsverbältnisse der Hornhautlamellen in den verschiedenen Schichten, bloss in den allermittelsten Corneallagen ein unmittelbarer Wundverschluss erfolgt während nach oben gegen die Bowman'sche Membran und nach unten gegen die Descemetis hin ein Voneinanderweichen der durchschnittenen Lamellen stattfindet, wie seinerzeit auch bereits schon Gussenbauer bei seinen Thierexperimenten hervorgehoben und auch Becker an der nach der Daviel'schen Methode angelegten Lappenwunde dargelegt hat. Nach oben und nach unten von dieser unmittelbaren Aneinanderlagerung bildet sich somit ein oberer etwas engerer und tieferer, und ein unterer etwas breiterer und kürzerer Wundtrichter, deren Tiefe ein Dritttheil der Hornhautdicke in der Regel übertrifft. (Fig. 4.) 
Der obere Wundtrichter wird nun eben in seiner ganzen Breite und Tiefe von der Epithelneubildung ausgefüllt; es wird dieser letzteren also dort die unterste Grenze gesetzt, wo die durchschnittenen Cornealfasern unmittelbar aufeinanderstossen.

Etwas modificirt werden diese Verhältnisse durch unsere gebräuchlichen Operationsmethoden. Bekanntlich ändern wir aus bestimmten Gründen die Ebene unserer Messerführung bei Ausführung sowohl der Iridectomie als auch der Extraction. Steil setzen wir das Lanzenmesser auf, um dann, nach geschehenem Einstiche, demselben durch Senkung des Heftes eine andere Richtung zu geben. Dem enisprechend erscheint denn auch der Wundcanal auf dem Durchschnitte bogenförmig oder gar wịnkelartig gekrümmt und erst anterhalb dieses Winkels findet die unmittelbare Aneinanderlagerung der durchschnittenen Corneallamellen statt. Je nach der höheren oder tieferen Lage dieses Winkels, der friiheren oder späteren Aenderung der Schnittebene also, wird der den oberen Wundtrichter verschliessende Epithelzapfen, je nach Umständen abgerundet oder fein zugespitzt, mebr oder weniger tief in die Cornea hineinragen, mitunter wohl bis an die Mitte derselben und tiefer.

Der etwas complicirteren Schnittfuhrung bei Anlegung unserer gebräuchlichen Extractionswunden vermittels des Graefe'schen Linearmessers entspricht auch eine verschiedene Profilansicht des Wundcanales an verschiedenen Längepunkten der Wunde, und demgemäss auch ein etwas ungleiches Verhalten des dieselbe verschliessenden Epithelzapfens an diesen differenten Stellen.

Als Untersuchungsobject für diese Verhältnisse diente mir ein durch Becker vollkommen lege artis extrahirtes und ohne Zwischenfälle geheiltes Auge einer plötzlich ver- 
storbenen sechzigjährigen Frau. An dem Einstichspunkte dieser Wunde ragt das Epithel als feiner, sich scharf zuspitzender Zapfen in den sehr schrägen und fast geradlinigen Wundcanal hinein, just bis an die Mitte des Hornhautdurchmessers, woselbst die durchsehnittenen Lamellen unmittelbar sich aneinander legen.

Etwas weiter von dem Einstichspunkte, gegen die Mitte der Wunde hin, und an der Stelle ungefähr, wo man der Messerklinge aus der dem Scleralborde ungefähr parallelen Ebene die Wendung nach vorne giebt, und wo also dem entsprechend der Wundcanal einen gekrümmten Verlauf nimmt und einen Winkel, und zwar gerade in der Mitte des Cormeadurchmessers besitat, - ist auch die Profilansicht des Epithelkeils eine andere geworden: er erscheint hier als schon beträchtlich breiterer und dabei leicht s-formig gekrümmter Zapfen, der nach unten auf den Winkel im Wundcanale mit leicht abgestumpfter Spitze aufstösst, nach oben mit seiner Basis sich gegen die Conjunctiva wendet.

Die darauffolgende Profilansicht des Epithelstocks ist auf Fig. 3. wiedergegeben. Bei Ausführung der Enucleation an der Leiche ist die offenbar noch recht zarte Wunde von unten nach oben gesprengt worden, was dem Studium derselben übrigens weiter keinen Abbruch that.*) Wir haben hier einen soliden Epithelzapfen mit breiter Basis vor uns, der allmahlig und scharf sich zuspitzend, senkrecht in die Cornealsubstanz und wohl bis über die Half te ihres Dickendurchmessers in diese hineinragt, bis an jene Stelle nämlich wieder, wo die Schnittfübrung aus der zu den Lamellen schrägen in die senkrechte übergeht, wo also eine Inanderfalzung der winkelig durchschnittenen Hornhautlamellen erfolgt.

*) Etwa drei Viertel des Hornhautdurchmessers sind auf der Zeichnung dargestellt, das untere Viertel nicht ausgeführt. 
Die schliesslich den mittelsten Parthien der Wunde entnommene Profilansicht zeichnet sich von der vorhergehenden dadurch aus, dass hier der Epithelzapfen sich nach unten noch feiner zuspitzt und noch tiefer, bis zu zwei D rittel der Hornhantdicke geht, oben jedoch an seiner Basis nicht vollkommen geschlossen erscheint, sondern eine erheblich tiefe, fein sich zuspitzende Einkerbung besitzt. Es verläuft somit in dieser Parthie, im Scheitel der corneal gelegenen Extractionswande und wo dieselbe also nach oben am meisten auseinanderklafft. im Epithelstock eine feine Rinne.

So bewahrheitet sich denn an dieser regelrecht angelegten und geheilten menschliehen Extractionswunde der oben aufgestellte Satz rollkommen, dass nämlich das Epithel allemal und jederzeit so tief in die Wunde hinabsteigt, bis es auf den natürlichen Widerstand der unmittelbar aufeinanderstossenden Hornhautlamellen stösst und dass infolge dessen Breite und Tiefe des epithelialen Verschlusses durch die Art der Schnittfübrung, den Aneinanderlagerungs- resp. Klaffungsmodus der Wunde bedingt werden.

Etwas anders allerdings liegen die Verhältnisse dort, wo Schnittführung und Aneinahderlagerung der Wandränder nicht derartig regelrechte sind, - wo die Wunde stärker klafft und es daher an keinem Punkte in dem Gesammtverlaufe des Wundeanales zu einem unmittelbaren Aufeinanderstossen der durehschnittenen Lamellen kommt. So sind es beispielsweise bei nicht ganz glatter Schnittführung bloss einzelne sich überschlagende Hornhautfasern, welche der epithelialen Wucherung von oben her die unterste Grenze setzen. - Ein noch weiterer Klaffungsgrad ist auf Fig. 2 vergegenwärtigt: nirgends kommt es hier zu einer unmittelbaren Berührung der durchtrennten Hornhautlamellen oder -Fasern. Die engste 
Stelle eines solchen, durchaus senkrecht gegen diese letzteren angelegten Wundeanales pflegt sich allemal unterhalb der Mitte des Hornhautdurchmessers zu befinden, während nach oben und nach unten von dieser Stelle die durehschnittenen Lamellen beträchtlich divergiren. Durch gegenseitige Einwirkung von Parenchymsaft und von Iristranssudat, sowie durch Stauung hier an dieser engsten Stelle, kommt es hierselbst zur Bildung eines die Wunde von hinten her versehliessenden oder verstopfenden Gerinnungshäutchens (a) oder -Pfröpfchens, hart bis zu welchem denn die epitheliale Wucherung von oben oder vorne vordringt, um sich hierorts aufzuballen.

\section{Die Karyokinese.}

Ist nach obiger Darstellung der die frische Hornhautwunde verschliessende Epithelzapfen nicht als ein bloss mechanisches Resultat, sondern als das Ergebniss eines Neubildungsprocesses aufzufassen; gehen wir von der Voraussetzung aus, dass Epithel eben nur aus Epithel gebildet werden könbe und erachten wir in den karyokinetischen Figuren den Ausdruck einer vor sich gehenden Zellenproliferation, so könnte der Nachweis soleher Figuren uns uber die Bildungsstätte des Epithelzapfens, sowio über den feineren Hergang bei Zustandekommen dieses Wundverschlusses einige Aufklärung geben.

Zum Zwecke der Darstellung karyokinetischer Figuren wurden die frisch enucleirten Kaninebenaugen sofort auf 18-24 Stunden in eine $1 / 10$ procentige, und dann für 2 Tage in eine 1/4 procentige Chromsäurelösung gebracht; nach der üblichen Härtung in Müller'scher Flüssigkeit, zweitägiger Wasserdurchspülung und successiver Alkoholbehandlung, in Celloidin gebettet und mit dem Microtom die Cornea senkrecht zur Wunde und stets in ihrer ganzen Länge geschnitten. Die Dicke der Schnitte durfte nicht mehr als $0,020 \mathrm{Mm}$., höehstens $0,025 \mathrm{Mm}$. 
betragen. Als Tinctionsmittel wurden Alauncarmin und Hämatoxylin angewendet. Wegen der grösseren Lebhaftigkeit des Processes erwiesen sich die centralen Linearmesserwanden als die für die Beobachtung günstigeren objecte, und begann diese letztere von den ersten Stunden nach ausgeführter Operation an.

Es erwies sich nun, dass karyokinetische Figuren sich am reichlichsten in der vierten Stunde nach der Verletzung nachweisen liessen, jedoch nicht etwa an dem Rande der Wunde angehäuft, sondern über die Gesammtoberfläche der Cornea verstreut. Schnitt für Schnitt fanden sich hier 8-16 Kerntheilungsfiguren in den mittleren und tieferen Schichten des Hornhautepithels, am reichlichsten in den Eusszellen*).

Die Disposition dieser Figuren über der Hornhautoberfläche ist auf Fig. 5 nach einem meiner Schnitte halbschematisch dargestellt. Bei w befindet sich die central angelegte Wunde, deren Ränder eben nur erst von dem durch doppelten Contour angedeuteten hineinwuchernden Epithel e umgriffen sind. Die Punkte $k, k, k$ in demselben bezeichnen die Oertliehkeit der Kerntheilnngsfiguren in dem natürlichen Verbältnisse ihres gegenseitigen $\mathrm{Ab}$ standes, während $\mathrm{K}, \mathrm{K}, \mathrm{K} \ldots$ die betreffenden Figuren selbst bei Zeiss F. Oc. 3 darstellen. Schon hier in diesem einen Schnitte sehen wir die karyokinetischen Figuren in reicher Mannigfaltigkeit der Stadien und Zwischenstadien, von der typischen anfänglichen Knäuelform des MutterKerns (K1), der Kranz- $\left(\mathrm{K}_{3}\right)$, der Stern- $\left(\mathrm{K}_{5}, \mathrm{~K}_{6}\right)$ Figur und der Aequatorialplatte $\left(K_{8}\right)$ bis $\mathrm{zu}$ dem Doppelstern des Tochterkerns (K9).

Doch das Wesentliche für uns liegt in dem Vertheilungsmodus dieser Figuren. Sowohl nach rechts

*) Normalitex lässt sich in dem Epithel der Kaninchencornea nach Vossius ungefähr eine solche Figur im Schnitte finden. 
als nach links von der Wunde findet sich die erste Kerntheilungsfigur erst in einem gewissen Abstande vom Wundrande. Darauf finden sich nach rechts $z u$, verhältnissmässig ziemlich dicht aneinandergereiht, zehn soleher Figuren, wäbrend der übrige Theil der Cornea bis zur äussersten Peripherie hin frei bleibt - bis auf eine einzige Figur, ungefabr in der Mitte. Nach links von der Wunde erscheinen die Figuren überhaupt spärlicher es sind deren bloss fünf vorhanden; doch auch diese bleiben nur auf eine gewisse Localität beschränkt und reichen kaum bis über die Mitte zur äussersten Peripherie der Hornhaut hinaus.

Nach diesem einen Schnitt za urtheilen, müsste anch für die in der Hornhaut central angelegte Schnittwunde dasselbe Gesetz gelten, das Eberth*) für die rundlichen Epitheldefecte in der Cornea, Leber**) für diejenigen in der vorderen Linsenkapsel fand, und wonach die Deckung eines solchen Defectes durch Vermittlung eines karyokinetischen Processes in einer Regenerationszone stattfindet, welche sich in einem gewissen Abstande von dem Defecte befindet. Doch die genaue Durchsicht einer grösseren Anzahl von Schnitten lehrt, dass für den vorliegenden Fall wenigstens dieses Gesetz in seiner ganzen wörtlichen Strenge wohl nicht zu nehmen ist: es treffen sich nämlich auch Schnitte, wo die karyokinestisohen Figuren, wenn auch spärlicher vorhanden - doch regellos über fast die ganze Hornhautoberfläche vertheilt erscheinen, - Schnitte, an denen sich solche Figuren bald dicht am Rande der Wunde, bald wieder an der fast äussersten Peripherie der Hornhaut ziemlich reichlich nachweisen lassen. Dennoch gewinnt man durch die Combination einer grösseren Anzahl von Schnitten die

*) Vifch. Arch. Bd. LXXVII.

**) Heildelberger Bericht 1878. 
Anschauung, dass die Karyokinese in einem gewissen, allerdings recht ausgedehnten Umkreise um die Wunde herum wohl lebhafter und reger vor sich gehe, als unmittelbar an dieser letzteren und in der äussersten Hornhautperipherie.

Verfolgt man den karyokinetischen Process zeitlich nun weiter, so ergiebt sich, dass derselbe in der zehnten Stunde nach Anlegung der Wunde bereits weniger lebhaft von statten geht, sowie dass nun jegliche Andeutung einer zonulär en Anordnung sich verloren hat. Man zählt jetzt bis zehn Figuren im Schnitt, in ziemlich weiten Abständen von einander, dem Wundrande mitunter wohl recht nahe, nicht selten aber auch wieder an der dussersten Hornhautperipherie, im Limbus conjunctivae selbst.*)

In der $\mathrm{z}$ wölften Stande nach geschehener Verwundung fanden sich die karyokinetischen Figuren zuers.t in dem Epithel der Wunde selbst, das nun schon beträchtlieh längs den Wandungen der letzteren hinabgesticgen war (Fig. I a und b) - in freilich nur beschränkter Zahl -1 bis 2 Figuren hin und wieder im Schnitt. Ebenso fanden sich 1 bis 2 solcher Figuren auch in einer 15stündigen Wunde - während aber gleichzeitig bis zu 10 Figuren über die Gesammtoberfiache der Hornhaut verstreut waren.

Hierauf aber klingt der karyokinetische Process auf der Hornhautoberfläche allmählig $a b$; $z$ war trifft man wohl auch nach 20 stunden nach Anlegung der Wunde bis zu 10 Figuren auf dieser Oberfläche, jedoch nach 24 Stunden sind dieselben bereits fast verschwunden.

Dagegen scheint ies, wie wenn jetzt, am Schlusse

*) Es wirde dieses Bild ungefähr mit den Angaben Robinsky's (Berl. Klin. Wochenschrift 1886 No. 39) hinsichtlich der vorderen Linsenkapsel übereinstimmen, der bei Verwundungen der letzteren die Kerntheilungsfiguren auch über die ganze Fläche ihres Epithelbelages zerstreut fand. 
des ersten und zu Beginn des zweiten Tages der Kerntheilungs-Process in der Wunde selbst ein lebhafterer wird: es finden sich hierselbst in dieser letzteren bis zu. 3 Figuren in Schnitt, wiederum in den tieferen und tiefsten Epithelschichten; ausserdem aber noch mehrfach verstreut kleine Zellen mit kleinem aber dunkel gefärbtem Kerne, einzeln oder zu $z$ weien nebeneinander, die wohk als junge, soeben aus der Theilung hervorgegangene Zellen anzusehen sind. In den späteren Stunden und Tagen beobachtet man wohl noch einzelne oder mehrere von den Kerntheilungsfiguren hin und wieder sowohl in der Wunde selbst, als in der Nachbarschaft derselben oder weiter auf der Hornhautoberfläche, doch der eigentliche Neubildungsprocess im Epithel ist jetzt als abgeschlossen zu betrachten.

Suchen wir uns nun nach den erhaltenen Bildern eine Vorstellung über den Hergang selbst in der Gesammtausdehnung des Epithels zu machen, so ist derselbe folgendermassen zu denken:

Allenthalben anf der Oberfläche der Hornhant, wo eine Kern- und Zellentheilung im Epithele vor sich geht, findet gleichzeitig eine Verdrängung und Verschiebung der benachbarten Epithelzellen statt, während der ersten Stunden nach der Verletzung in der äussersten Hornhautperipherie weniger, in einem gewissen Umkreise um die Wunde herum in lebhafterer Weise, - allemal aber in der Richtung gegen diese letztere hin. Allmälig schiebt sich denn auf diese Art das Epithel von der Oberflache um den Rand der Wunde herum und in diese letztere hinein, indem es längs der Wandungen derselben hinabgleitet. Ist dieses bis zu einem gewissen Grade geseheben, so wird ein weiteres Hinabgleiten in die Tiefe auf zweifache Weise veranlasst: einerseits durch ein weiteres Vorschieben des Epithels der Hornhautoberfäche Dank der Fortdauer des karyokinetischen Processes auf dieser letzteren, 
andererseits endlich durch eine ebensolche Verdrängung und Verschiebung von Zellen in dem Epithelhäutchen der Wunde selbst, indem auch hier jetzt Kern- und Zellentheilung in eingestreater Weise vor sich geht (Fig. I. a), wobei das Wachsthum durch Apposition an dem freien Ende des Häutchens (wie z. B. bei b) wohl nur hin und wieder vorzukommen scheint. So geht es weiter, bis der natürliche Widerstand in dem Wundcanal erreicht, und die freien Enden des beiderseits herabgeglittenen Epithels an diesem Orte aufeinanderstossen.

Doch hiermit ist die producirende Kraft des Epithels der Hornhautoberfiäche noch nicht erschöpft. Immer dauert noch auf dieser die Karyokinese fort und immer noch schiebt sich von hier aus Epithel in die Wunde hinein und in dieselbe hinab, um sich an dieser. Stelle zu einem Ballen (d) aufzurollen (Fig. 2). Auch in diesem letzteren findet indirecte Kerntheilung noch statt (c), wodurch es zu einer concentrischen Vergrösserung desselben kommen kann, während er selbst durch einen gleichzeitigen derartigen Process in der unter ihm befindlichen Epithelbrücke auch noch emporgehoben wird, ohne dass jedoch in einigermassen stärker klaffenden Wunden das eigentliche Niveau der Hornhautoberfläche erreicht zu werden pflegt.

Ueberhanpt muss man sich nicht der Vorstellung hingeben, es sei die epitheliale Neubildung, einmal vorhanden, auch im Stande eine jede noch so weit klaffende Wunde so zu sagen bis an den Rand vollkommen auszufüllen. Nur in kleineren und weniger klaffenden Wunden, an den Winkeln der Extractionswunde, ist dieses der Fall. Hier, wo der Klaffungsgrad der obersten Wundoffnung jedenfalls nicht breiter als die doppelte Höhe der Epithellage auf der Hornhautoberfläche ist, erfolgt ein sofortiges Aufeinanderstossen der durchschnittenen und in die Wunde beiderseits sich hineinschiebenden Epithelien; gegenseitig drücken sich die Zellen gegen einander $a b$ und 
bilden sofort einen von der Basis bis zur Spitze soliden Zapfen. Etwas anders schon verhält es sich bei der auf Fig. 3 abgebildeten Parthie der normalen Extractionswunde Hier überschreitet die Breite der obersten Wundoffnung die doppelte Höhe der Hornhautepithelien bereits um ein Beträchtliches und befindet sich infolge dessen der Berührungspunkt dieser in der Wunde in einer schon beträchtlichen Tiefe. Doch durch das auch hier, just wie bei der Kaninchenwunde Figur 2, sich von der Hornhantoberfläche noch hinterher nachschiebende Epithel, wird auch hier eine ballen- oder kugelförmige Aufrollung dieses letzteren (e) mit zwiebelschalenartiger Anordnung der Zellen, oberbalb des erwăhnten Beruhrungspunktes, sowie eine solide Ausfüllung der Wundöffnung veranlasst. $\mathrm{Ob}$ nun die Lage dieser Kugel die ursprüngliche ist, oder $o b$ dieselbe durch poliferative (karyokinetische) Vorgänge in der Tiefe der Wunde bereits emporgehoben worden ist, lässt sich jetzt, am 9. Tage, wo der karyokinetisehe Process längst abgeklungen, wohl kaum noch feststellen.

Offenbar noch tiefer liegt der Berührungspunkt der beiderseits hinabgeglittenen Epithelien in den am meisten klaffenden, mittelsten Parthien der Extractionswunde; zu einer Aufrollung eines für den betreffenden Raum übermächtig reichlichen Zellennachschubes von der Hornhautoberfläche her, ist es in dem vorliegenden Falle, wenigstens an dieser Stelle, nicht gekommen, allenfalls vielleicht, dass durch eine Zellenproliferation in der Tiefe der Wonde die ursprüngliche Oberfläche des Epithelhäutchens hierselbst noch um ein gewisses emporgehoben worden ist; doch hiermit ist die Epithelwucherung auch abgeschlossen, denn nach oben zu bleibt ein, wenn auch schmales, so doch ziemlich tiefes Lumen zurück, das selbst nach 9 Tagen sich noch als feine Rinne in dem Epithelstock präsentirt.

Stets zu einem vollkommen soliden Epithelverschluss hingegen kommt es bei der gewohnlichen Lanzenmesser. 
wunde, an der sich nach zwei Tagen ein etwaiges zurückgebliebenes Lumem niemals nachweisen liess. Trichterartig schiebt sich hier zuerst das Epithel der Hornhautoberfläche in die Wundöffnung hinab, um dann, nach gegenseitigem Berühren und Confiniren der freien Endungen durch weiteres Nachschieben sowie durch Zellenproliferation in der Tiefe der Wunde selbst emporgehoben and dem übrigen Hornhautniveau vollkommen gleich gemacht zu werden.

Allemal aber scheint der karyokinetische Process nur in den tieferen und tiefsten Epithelschichten vor sich zu gehen, derartig dass die Ausfüllung des Wundtrichters stets durch Verschieben, Vorrücken und Emporheben einer bereits vorhandenen Epitheloberfläche erfolgt.

\section{Vernarbung und Ausstossung.}

Verfolgt man nun den Heilungsvorgang in dem $\mathrm{Ge-}$ sammtverlaufe einer Lanzenmesserwunde, so ergiebt sich Folgendes:

Sofort nach Anlegung einer solchen Wunde beginnt eine Aufquellung der Hornhautlamellen, sowie eine kolbenartige Anschwellung ihrer freien durchschnittenen Enden, durch Einwirkung des vorströmenden Kammerwassers bedingt. Alsdann findet ein zahnartiges Ineinandergreifen der mittelsten Corneallamellen, deren Elastisität am geringsten and deren gegenseitiger Abstand nach der Durchtrennung daher auch der kleinste ist, sowie eine Verklebung derselben durch den hervorquellenden Parenchymaft statt. Es beginnt jetzt die Wiederherstellung der Vorderkammer, sowie die Ausfüllung des äusseren Wundtrichters durch das von oben hineinwuchernde Epithel, während der innere Wundtrichter sich durch ein Gerinnungspfröpfchen schliesst, durch gegenseitige Einwirkung von Parenchymsaft und von Iristranssudat entstanden. Nach 30 Stunden ist die Bildung des Epithelzapfens bekanntlich vollendet und der provis oris che Wundverschluss 
hiermit hergestellt (Fig. 6). Nur sehr massig sind in solch einer reactionslos heilenden Hornhautwunde und um dieselbe Rundzellen vertreten: bloss vereinzelt finden sich dieselben in den etwaigen Lücken des mittleren engsten Theils des Wundcanales, bloss in geringer Anzahl in dem Gerinnungspfröpchen des inneren Wundtrichters, in durchaus nicht reichlicher Weise über die benachbarte Hornhautsubstanz verstreut, und allenfalls nur in der Gegend des Limbus conjunctivae lässt sich ein dichterer Zellenschwarm bemerken. Das Hornhautparenchym selbst erscheint um das Doppelte bis Dreifache seines normalen Durchmessers in weiterer Umgebung um die Wunde geschwellt; die Hornhautlücken sind beträchtlich erweitert, die fixen Zellen in denselben beträchtlich gequollen. In den tiefsten Hornhautschichten ist dieses weniger als in den oberflächlichen der Fall. Die durchschnittenen Enden der Membrana Deseemetii weichen weit von einander, ihre Einbiegung gegen die Wunde hin ist eine nur höchst unbedeutende. An dem Endothel der Descemetii liessen sich keine Veränderungen wahrnehmen.

In diesem Stadium des provisorischen Versohlusses, der also durch mechanisches Ineinandergreifen resp. Verklebung der mittelsten Corneallamellen, durch das Gerinnungspfröpfchen nach innen und den Epithelzapfen nach aussen gebildet wird, und wobei dieser letztere wohl die solideste Verbindung darstellt, verharrt die Wunde ziemlich unverandert zwei Tage lang. Erst am dritten Tage lässt sich eine Veränderung in dem Sinne wahmehmen, als eine reichlichere Ansammlung von Rundzellen unmittelbar am Wundcanale, und längs seiner ganzen Ausdehnung, beginnt.

Der eigentliche Tag der Entzundung ist jedoch der vierte Tag. Man kann diese letztere mit Recht als eine reactive bezeichnen, indem dieselbe nicht nur den definitiven Wundverschluss, sondern auch die Ausstossung 
des Epithelzapfens und Ersetzung desselben durch Bindegewebe bezweckt. Mächtig sind jetzt (Fig. 7) die interlamellären Lücken erweitert, durch Rund- und Spindelzellen, die sich kettenartig aneinanderreihen, förmlich vollgepfropft. Auch der Wundcanal selbst in seinem mittleren, engsten Abschnitte, sowie der innere Wundtrichter sind jetzt von solchen Zellen reichlich angefüllt. Unmittelbar um den Epithelzapfen aber hat sich eine förmliche Zone von Rund- und Spindelzellen gebildet, wie etwa die Demarcationslinie um einen Fremdkörper oder um eine necrotisirte Gewebsparthie. Aus den benachbarten Parenchymlücken scheinen die namentlich zahlreich vertretenen Spindelzellen gleichsam hervorzuschwimmen, den oberen, stumpferen Abschnitt des Epithelzapfens zu umfassen und von seiner Umgebung zu lockern, in seiner Mitte ihn za durchsetzen und seinen untersten, spitzen Antheil von ihm abzuschneiden. Auch in diesen untersten Abschnitt dringen die Spindelzellen theilweise zwischen die Epithelien ein, theilweise umschliessen sie denselben ringförmig yon aussen.

Es sind bei diesem ganzen Entzündungsprocesse aber die Rund- und Spindelzellen zum Theile mindestens als Abkömmlinge der fixen Hornhautzellen zu betrachten, indem sich an diesen letzteren karyokinetische Figuren, und das zwar in der nächsten Nachbarschaft der Wunde, mit Sicherheit nachweisen lassen (Fig. 10), wie solches auch bereits von Homèn*), der unter der Leitung Cohnheim's arbeitete, bei der Regeneration ron Substanzverlusten in der Cornea, von Klemensiewicz **) bei Aetzungen der letzteren behauptet worden ist.

Nach weiteren 24 Stunden, am fünften Tage nach Anlegung der Lanzenmesserwunde, ist das erste acuteste

*) Fortschritte der Medicin, 1. 16.

**) Centralbl. f. d. med. Wiss. 1884. 
Stadium der reactiven Entzündung bereits vorüber: die Schwellung des Hornhautparenchyms hat beträchtlich abgenommen, die Rund- und Spindelzellen in den Parenchymlücken sind fast verschwunden. In der den Epithelzapfen umgrenzenden Demarcationszone haben die Spindelzellen sich in die Länge gezogen, haben eine festere Consistenz angenommen, sind näher aneinander, dichter an das Epithel herangerückt; den inneren Wundtrichter erfüllen sie jetzt vollkommen, indem sich Zelle dicht an Zelle schliesst.

Am sechsten Tage nach der Verwundung (Fig. 8) sind die Spindelzellen um das Mehrfache ihrer ursprünglichen Länge ausgewachsen. Durch theilweise faserige Umwandlung und Retraction des neagebildeten Bindegewebes wird jetzt ein Druck auf das Epithel ausgeübt: der untere Abschnitt des Epithelzapfens (a) wird einfach umschnürt, die Zellen desselben fallen späterhin der Atrophie anheim und gehen auf diese Weise zu Grunde. Der obere Antheil (b) wird theilweise seitlich comprimirt, theilweise von unten nach oben emporgehoben. Es findet infolge dessen jetzt eine palissadenartige Anordnung der allmälig Cylinderform annehmenden seitlichen und untersten Begrenzungsepithelien statt, während die obersten Epithelschichten, über das gemeinsame Niveau emporgehoben und aufgethürmt (c), einer Degeneration anheimfallen und allmälig abgestossen werden.

Einer Degeneration vereinzelter Epithelien, inmitten des Zapfens verstreut, begegnen wir bereits in den allerersten Tagen nach der Verwundung. Es erscheinen diese Zellen vergrössert und von sphärischer Gestalt, mattglänzend, durch Alauncarmin und Eosin (nach vorherigem Aufenthalt der Präparate in Chromsäure) nicht gefärbt, die unveränderten Reste des Protaplasma nebst Kern am Rande der Zelle, so dass die typische ,Siegelring"-Figur entsteht (Fig. 6,a), oder es ist eben nur noch die Spur eines randständigen Kernes zu unterscheiden (Fig. 7, a). - 
Weiterhin schwindet der Kern vollkommen (Fig. 7, b) und es erfolgt nun ein "Zusammenschweissen" oder "Versintern" mehrerer benachbarten Zellen (Fig. 7, c), wie v. Recklingshausen*) solches für die hyaline Degeneration, Weigert**) - für die byaline Coagulationsnecrose, als eigenthümlich erachtet.

In den der Oberfläche zunächst gelegenen oder derselben aufgelagerten Epithelien erscheint ter Kern vor allem abgeblasst (Fig. 6, b), mitunter kaum nur noch angedentet oder es fehlt derselbe rollkommen (Fig. 8 , bei c); das Protoplasma wie mit einem Locheisen durchschlagen, mitunter bis auf einen nur feinen Ring an der Zellenperipherie reducirt, oder aber in seiner Gesammtheit in eine mattglänzende, homogene, glasige, ungefärbt bleibende Masse umgewandelt; die Gesammtzelle gequollen, rundlich oder eiförmig oval, mitunter mächtig erweitert (d); die einzelnen Zellen entweder noch in gegenseitigem Zusammenhange oder schollenartig sich übereinander schiebend.

Es wäre diese Oberflächendegeneration, soweit sich an den in Chromsäure und Müller'scher Flüssigkeit gelegenen Präparaten entscheiden lässt, als schleimige oder aber auch als hyaline zu deuten. Ich glaube dieselbe, der vielfachen Uebergänge halber, aber jedenfalls mit derjenigen in den tieferen Schichten des Epithelzapfens identificiren und die gesammte Degeneration als eine hyaline bezeichnen za dürfen, - am Richtigsten vielleicht in diesem Falle im Sinne Weigert's als hyaline Abart der Coagulationsnecrose aufzufassen, für deren Zustandekommen die nothwendigen Bedingungen mir hierselbst durehaus vorzuliegen scheinen.***)

*) Deutsche Chirurgie.

**) Deutsche Medicinische Wochenschrift 1885 No. 44.

***) Einer persönlichen Mittheilung Herrn Prof. Arnold's zufolge, der die Freindlichkeit hatte, meine Präparate zu durchmustem, erinnert der gesammte Vorgang selr an die Epithel- 
Es spielt diese Art ron Degeneration in dem gesammten Wundheilungsverlaufe eine Rolle. Wir begegnen ihr bereits am zweiten Tage, wo ihr die Epithelien der Oberfläche überall dort anbeimfallen, wo eine Ueberproduction von Zellen in der Tiefe stattfindet. Es ist das namentlich um die 24. bis 30. Stunde der Fall, wo der endgültige Versehluss des neugebildeten Epithelzapfens vor sich geht und durch gleichzeitiges Aufeinanderstossen von Fon den beiderseitigen Hornhautoberfächen und aus der Tiefe des Wundtrichters vorrückender and sich dem gegegebenen Raume anpassender Zellen eine gegenseitige Compression und Abstossung äberschussiger an dem Verschlusspunkte stattfinden muss. Die Localitat dieser Degeneration in und an dem sich schliessenden Lumen, sowie der Umstand, dass ihr um diese Zeit fast ausnahmslos die Epithelien der Oberfläche anheimfallen, sprechen auch fur die Thatsache, dass der Epithelverschluss zuerst durch Verschiebung von der Hornhantoberfläche aus, darauf durch Aufrücken von unten her herbeigeführt wird.

An den folgenden Tagen des Stillstandes finden sich die degenerirten Zellen nicht mehr an der Oberfäche, sondern hin und wieder inmitten des Zapfens selbst verstreut. Gleichzeitig mit dem von Seiten des demarkirenden Bindegewebes aus auf den Zapfen ansgeübten Drucke nimmt ihre Anzahl zu und am fünften Tage erscheint dieselbe schon ziemlich reichlich, und zwar jetzt vornehmlich unterhalb der Epitheloberfläche.

Endlich bemerkt man am sechsten Tage eine ähnliche (hyaline) Degeneration auch an einzelnen dem Wundcanale benachbarten Wanderzellen.

Am a chten Tage (Fig. 9) erscheint der Epithelzapfen sowohl an seiner Oberflache als in seinem Innern von

veränderung bei Trennung der embryonalen Lidspalte (SehweiggerSeidel, Virchow's Arch. XXXVII; - Ewetzky, Archiv f.. Au;enheilkunde VIII). 
degenerirten Zellen vollkommen befreit. Er ist in seiner Grösse jetzt erheblich reducirt. Die tiefsten Zellen in ihm haben Gestalt und Anordnung der typischen Fusszellen angenommen, die mittleren sind regelmässig rundlich polygonal, die oberflächlichsten flachen sich allmälig ab. Das begrenzende Bindegewebe hat sich in eine ausgeprägte Faserschicht umgewandelt mit der dentlichen Tendenz durch fernere Organisation and Retraction ein weiteres Emporheben des Epithels zu bewirken. Der untere Theil des oberen Wundtrichters ist mit jungen Bindegewebszellen angefunlt, die hier ein deutliches Dreieck bilden; hier und gegen die mittelste Parthie des Wundeanales finden sich verstreute, theilweise atrophische und dem Untergange anheimgegebene Epithelien. Das benachbarte Hornhantparenchym ist, wenn auch weniger als früher, doch immer noch geschwellt, es findet noch immer eine, wenn auch mässige Zellenimigration von dort aus statt. In dem untern Wundtrichter geht mittlerweile eine faserige Umwandlung und netzartige Anordnung der daselbst angesammelten Spindelzellen einher.

In den folgenden Tagen erfolgt nun ein weiteres Vorrücken der sich organisirenden Bindegewebsschicht gegen die Hornhautoberfläche, eine Degeneration und Abstossung der oberflächlichsten emporgehobenen Epithelien und dem entsprechend ein successiver Ersatz durch Emigration friseher Wanderzellen von unten her, vornehmlich also aus den mittleren Hornhautschichten. Auf die Art ist denn am vierzehnten Tage das Epithel über der Wunde in der Weise ausgeglichen, dass es von dem benachbarten nach Anordnung and Dicke sich durchans nicht mehr unterscheiden lässt. Der obere Wundtrichter ist jetzt durch junges Bindegewebe ausgefüllt, dass durch die feinere Faserung, sowie durch die reichlichere Gegenwart von Zellen und ron Kernen als nach unten zugespitztes Dreieck sich deutlich genug von dem übrigen Hornhautparenchyme 
absetzt. Der centrale engste Theile des früheren Wandcanales tritt auch noch durch etwas reichlichere Ansammlung zelliger Elemente in seiner Nachbarschaft hervor. Der untere Wundtrichter ist durch ein etwas wirres Fasernetz geschlossen, in dem sich nur wenige Zellen nachweisen lassen.

Nach einer weiteren Woche, am dreiundz wanzigsten Tage nach der Verwundung, lässt sich der Verlauf der Wunde nur noch mit Schwierigkeit inmitten des normalen Gewebes entdecken. Entsprechend der centralen Parthie des Wundcanales findet jetzt ein vollkommen ununterbrochenes Hinüberlaufen der Lamellen statt und nur hier und $\mathrm{da}$, an der Stelle früherer, grösserer Lücken, lassen sich faserig ausgewachsene Spindeln von schräger, oder mehr weniger steiler Richtung wahrnehmen. Die Region des oberen Wundtrichters lässt sich nur dadurch einigermassen abgrenzen, dass die Lamellen hierselbst, im Vergleiche zu dem benachbarten Parenchyme, einen mehr gewellten Verlauf nehmen and sich an dieser Stelle eine Bowman'sche Membran nicht nachweisen lässt. Dor untere Wundtrichter tritt mit grösserer Deutlichkeit hervor: Die Fasern in ihm haben jetzt zwar auch eine mehr regelmässige lamelläre Anordnung genommen, besitzen aber eine gegen das Normale etwas schräge Richtung, erseheinen auch noch leicht gewellt, feiner und weiter von einander gerückt.

Am dreissigsten Tage endlich nach Anlegung der Lanzenmesserwunde, finden sich nur äusserst wenig Anhaltspunkte, um' die Oertlichkeit der äusseren Wundöffnung, zumal bei peripherer corneaseleraler Lage derselben, mit Sicherheit zu constatiren: das Epithel ist längst von durchaus normaler Beschaffenheit, die Nichtexistenz einer Bowman'schen Membran, der etwaige etwas gewelltere Verlauf der Lamellen ist an dieser Stelle nicht auffallend. 
Am längsten lässt sich noch der untere Wundtrichter abgrenzen, indem auch jetzt hierselbst die Lamellen eine noch leicht schräge Richtung, eine feinere Beschaffenheit besitzen. $\mathrm{Zu}$ einer eigentlichen Wiederherstellung der Membrana Descementii oder directen Verwachsung ihrer durschnittenen freien Einden kommt es niemals; es sind um das Mehrfache feinere Faserlagen, die diese letzteren miteinander verbinden. Eine etwas erheblichere Einrollung dieser durchschnittenen Enden ist auf Unregelmässigkeiten bei der Operation, gewaltsames Hineinzerren bei Ausführung der Irisexcission, auf abnorme Wundheilung zurïckzuführen.

Etwaiges Pigment, das bis zuletzt restirt und den Verlauf der einstigen Wunde markirt, ist durch mechanische Verschleppung resp. Abstreifung bei Ausführung der Iridectomie zu erklären.

Analog, wenn freilich im Einzelnen auch etwas unterschiedlich, werden sich die weiteren Schicksale des Epithelstocks in der Linearmesser-oder Extractionswande gestalten. Die Schwierigkeiten, eine wirkliche, ideale prima intentio einer derartigen etwas längeren Wunde beim Kaninchen zu erreichen, erschweren das systematische Studium derselben. In der uns vorliegenden normalen mensehlichen Extractionswunde (Fig. 3) bildet am neunten Tage nach ausgefürter Operation das Epithel mehr als zwei Dritttheile des gesammten Wundverschlusses, während der übrige Theil dieses letzteren durch bereits fester organisirte Spindelzellen bewirkt wird. Nichtsdestoweniger ist es bekannt, dass auch von einem derartig weit in die Tiefe hinabragenden Epithelverschlusse nach mehreren Monaten nicht eine Spur mehr vorhanden und derselbe durch Bindegewebe resp. normal verlaufende Corneallamellen vollkommen ersetzt ist. Offenbar, dass hier der 
Austossungs- und Vernichtungsprocess sehr viel langsamer und allmäliger von statten geht, als an der Lanzenmesserwunde des Kaninchens, theilweise wohl durch die verhältnissmässig grössere Tiefenausdehnung des Epithelkeils, theilweise durch die grössere Längenausdehnung der Wunde, theilweise endlich durch das hohe Alter des menschlichen Individuums und die demselben eigenthümliche grössere Resistenz und Persistenz epithelialer Gebilde bedingt. Auch erseheint es, bei der beträchtlichen Länge und verhälnissmässigen Schmalheit des Epithelzapfens wenig wahrscheinlich, dass ein grosser Theil desselben emporgehoben und in grosser Ausdehning einer Oberflächendegeneration anheimfällt. Wahrscheirilicher ist es wohl, dass an verschiedenen Punkten seiner Länge die. jungen Spindelzellen in ihn eindringen und mehrorts grössere and kleinere Epithelinseln nmringen, umschnưren und zur Atrophie bringen. Eine besondere Aufmerksamkeit nimmt die Perlkugel (e) anf unserer Figur in Anspruch, denjenigen bei einem Cancroide vollkommen analog. Man wäre aus diesem letzten Grunde leicht versucht diese Kugel mit dem endgültigen Zerfalle des Epithelzapfens in näheren causalen Zusammenhang zu bringen, wenn wir anderseits nicht wüssten, dass vollkommen analoge Kugeln oder Knoten sich häufig und reichlich in der Epidermis aiterer Individuen sowohl unter pathologischen als auch normalen Verhältnissen vorfinden, ohne dabei die geringste Neigung zu einem Zerfalle zu besitzen. Auch die Art der centralen Degeneration dieser Kugel, die der hyalinen auch wohl ähnlich, des dunkleren gelblichröthlichen Glanzes in dem Eosinpräparate halber, aber wohl eher als colloide aufzufassen ist, spricht jedenfalls gegen die Wahrsoheinlichkeit eines baldigen Zerfalls. Ich glanbe die Bildung dieser Perlknoten bereits in die ersten 24 Stunden nach Anlegung der Wunde zurückversetzen zu müssen, indem ioh voll- 
kommen analoge Gebilde um diese Zeit beim Kaninchen wiederholt beobachtete (Fig. 2), zuerst in der 15. Stunde, und dann anch später. Ihre Entstehung glaube ich, wie bereits angedeutet, durch Aufrollung sich von der Hornhautoberfläche in die Wunde hineinschiebenden Epithels erklären zu müssen. Ein constantes Vorkommniss scheinen sie auch nicht zu bilden und wohl durch versehiedene Umstände, unter anderem wohl auch die Klaffungsbreite und - tiefe des Wundcanales bedingt zu sein. Dass auch diese Kugeln, wie der gesammte Epithelzapfen, auf dem einen oder anderen Wege zuletzt auch dem Untergange anheimfallen, bleibt schliesslich selbstverständlich.

\section{Die abnorme Wundheilung.}

Je mehr die Wunde auseinanderklafft, um so mehr verliert das Epithel seine, die Wunde verstopfende und zusammenhaltende Bedeutung and erfüllt es jetzt nur die Bestimmung als äussere Schutzdecke für dieselbe. Denn wegen der in der Wunde selbst bloss beschräniten Zellenproduction, kommt es bei einem gewissen Klaffungsgrad derselben nicht mehr zur vollkommenen Ausfüllung ihres oberen Lumens, nicht mehr zur Bildung eines soliden Epithelzapfens, sondern bloss zur Bildung eines mehr oder weniger flachen trichterartigen Ueberzugs, der eine gewisse Mächtigkeit allerdings erreichen kann. Je grösser aber auch der Klaffungsgrad der Wunde, um so mehr findet Imigration ron Wanderzellen in dieselbe statt; je mehr also Wanderzellen, um so weniger verhältnissmässig Epithel. Je lebhafter die reactive Entzündung, um so eher kommt es aber auch zur Emporhebung und oberflachlichen Abstossung des neugebildeten Epithels. Ist endlich die Entzündung von Hause aus eine sehr lebhafte, so wird das neugebildete Epithelhäutchen durch die reichlich an- 
rückenden Wanderzellen durchbrochen, oder es sistirt gar die epitheliale Neubildung von Anfang an, sie tritt vor der bindegewebigen einstweilen rollkommen zurück.

Herrn Geheimrath Becker in Heidelberg, auf dessen Anregung und in dessen Laboratorium ich diese Arbeit ausgeführt, spreche ich hiermit meinen besten Dank aus. 


\section{Erklärung der Abbildungen.}

F ig. 1, Linearmesserwunde vom Kaninchen nach 12 Stunden. Bei a und $\mathbf{b}-$ Kerntheilungsfiguren (Zeiss F. Oc. 3).

Fig. 2, Linearmesserwunde desgl. nach 15 Stunden.

Bei $c$ - Kerntheilungsfigur. d - Epithelballen (Zeiss DD Oc. 3).

Fig. 3. Periphere Extractionswunde rom Menschen nach 8 Tagen.

e - Perlkugel (Zeiss DD. Oc. 3).

Fig. 4. Lanzenmesserwunde vom Kaninchen nach 3 Stunden.

F i g. 5. Linearwunde rom Kaninchen nach 4 Stunden mit karyokinetischen Figuren.

w - Wunde. e - Epithel. $k, k, k$ - Oertlichkeiten der Kernthellungsfiguren. $\mathrm{K}_{1}-\mathrm{Knäuelform.} \mathrm{K}_{2}-$ lockerer Knäuel. $\mathrm{K}_{\mathrm{3}}-$ Kranzform. $\mathbf{K}_{4}-$ Zwischenstadien von der Knẩuel- zur Sternform. $K_{5}-$ Sternfigur. $\quad K_{6}$ - feinstrahliger Stern. $K_{7}-$ Uebergänge von der Sternform zur Aequatorialplatte. $\mathbf{K}_{\mathrm{g}}$ - Aequatorialplatte. $K_{9}-$ Doppelstern des Tochterkerus (Zeiss F. Oc. 3).

F ig. 6. Lanzenmesserwunde vom Kaninchen nach 30 Stunden.

a - degenerirte Zelle mit randständigem Kern und Protoplasma. b - dito mit abgeblasstem Kerne.

Fig. 7. Lanzenmesserwunde vom Kaninchen nach 4 Tagen.

a - degenerirte Zelle mit randständigem Kern. b - ohne Kern. c - zusammengeschmolzene Zellen. 
Fig. 8. Desgleichen nach 6 Tagen.

a - unterer, abgeschnürter Antheil des Epithelzapfens. b - oberer Antheil. $\mathrm{c}$ - degenerirte kernlose Zellen. d - degenerirte mächtig erweiterte Zelle (Zeisś DD 0c. 3).

Fig. 9. Desgleichen nach 8 Tagen.

Fig. 10. Stabile Hornhautzellen mit karyokinetischen Figuren. ( $Z$ eiss, F. Oc. 3.) 
6

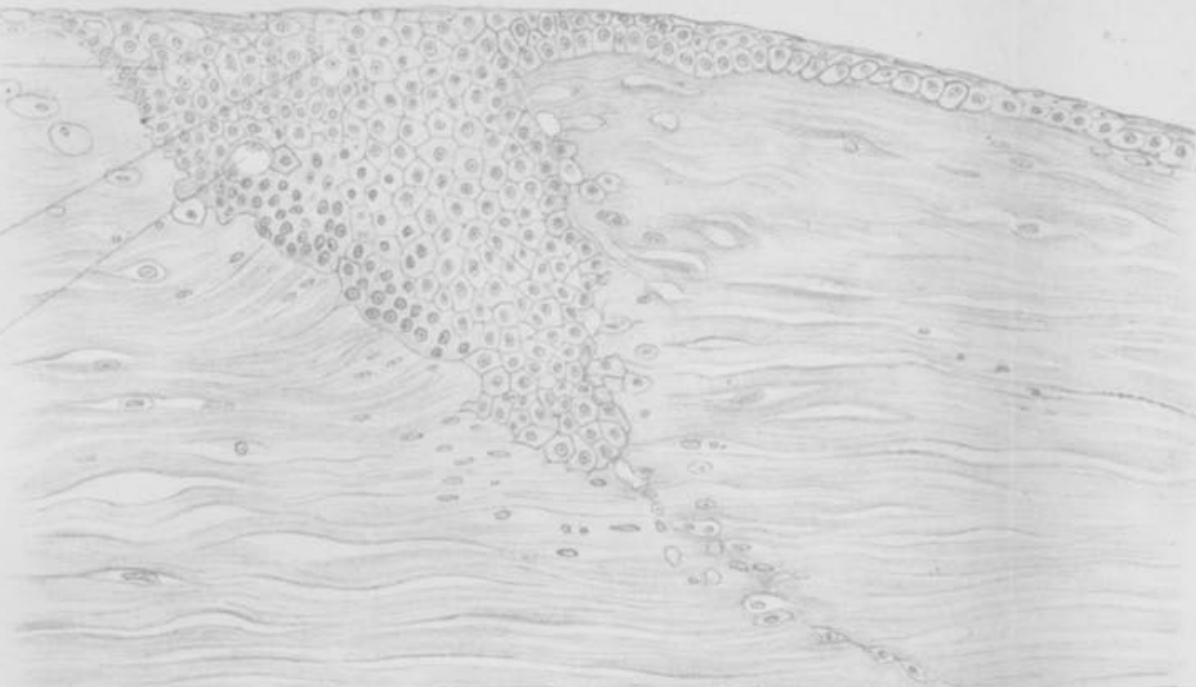

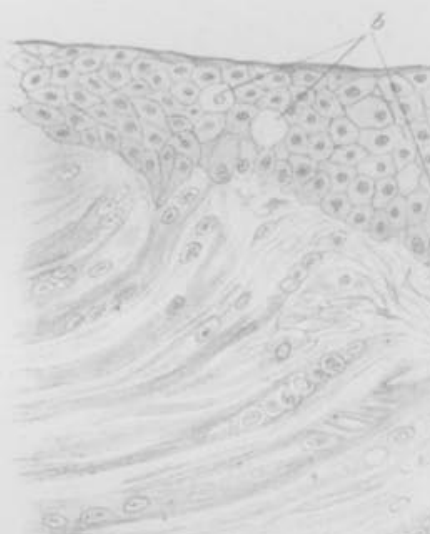

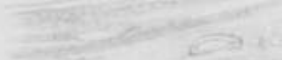

\section{acosn}

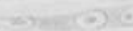

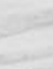

(क) लxक्षणतने

O. QPekero aro

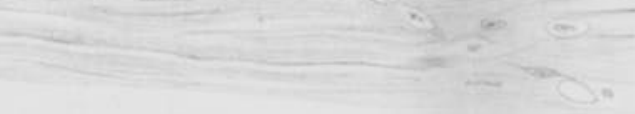 \\ Fig 7}

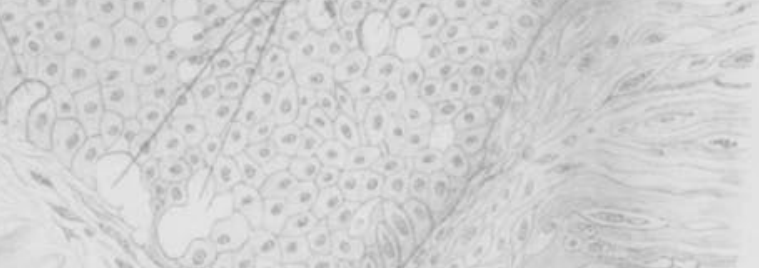

A

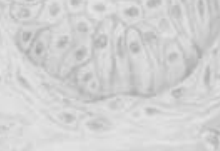

(4)

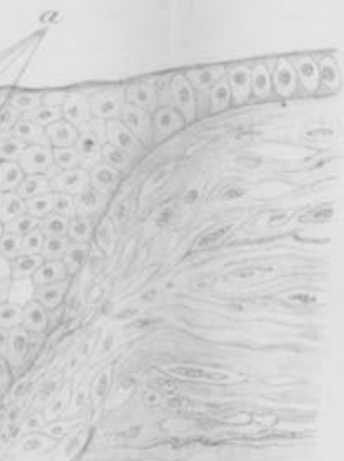

$\sqrt{8}$ 


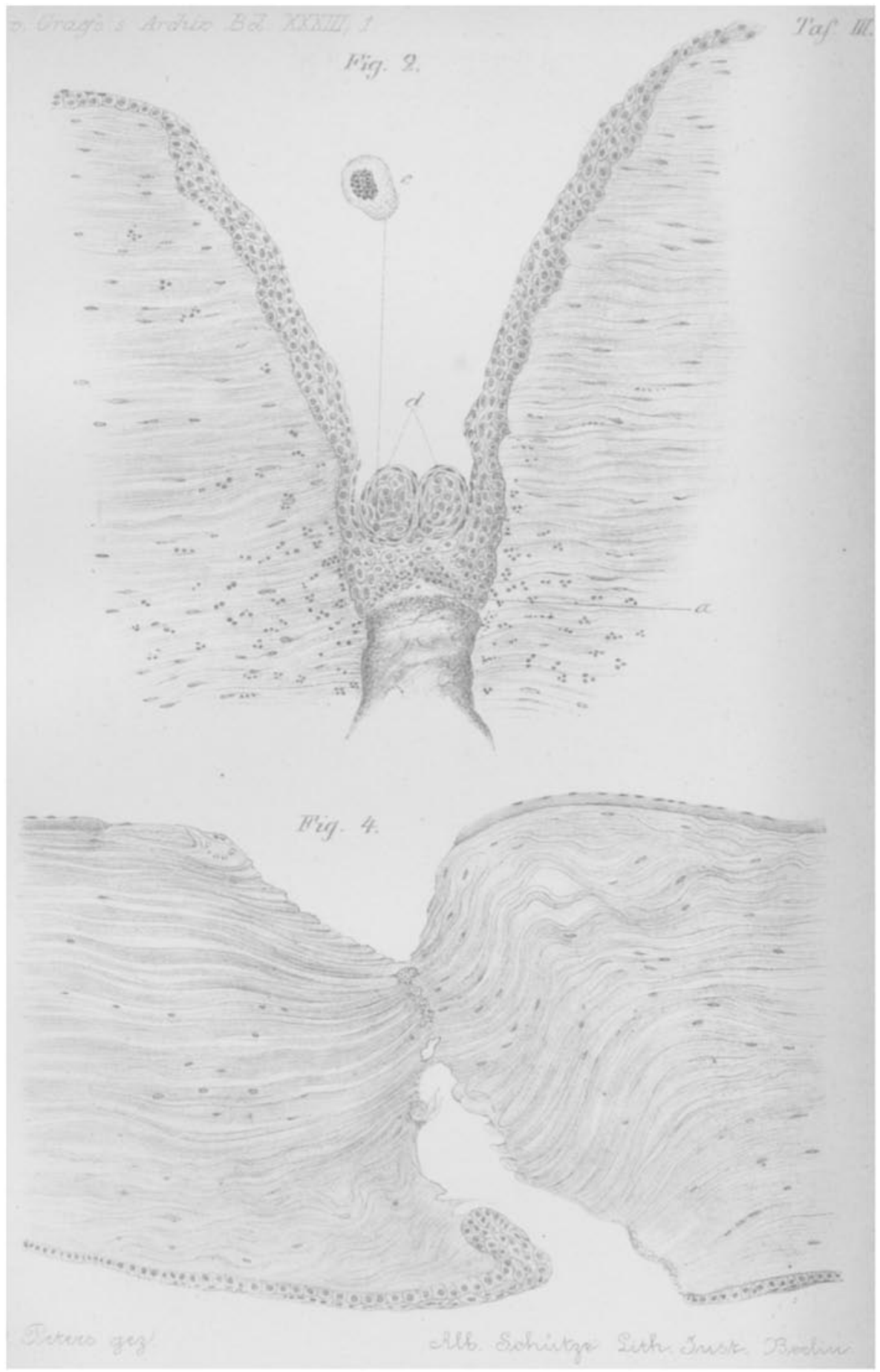




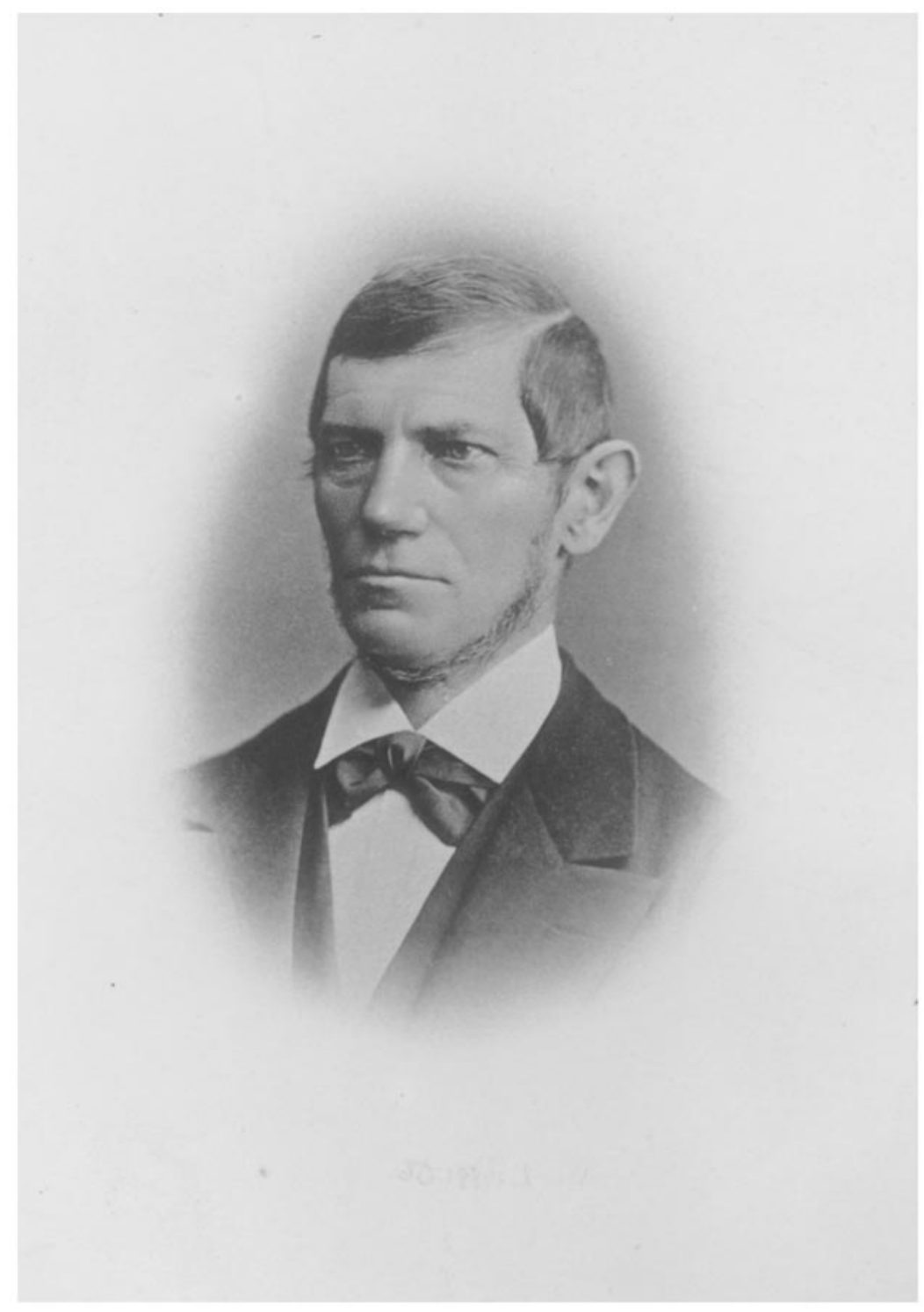

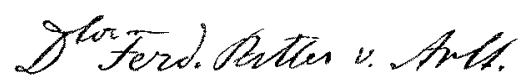

Verlag ఛ. Wilh. ENGELMANN, Ieipzig. 


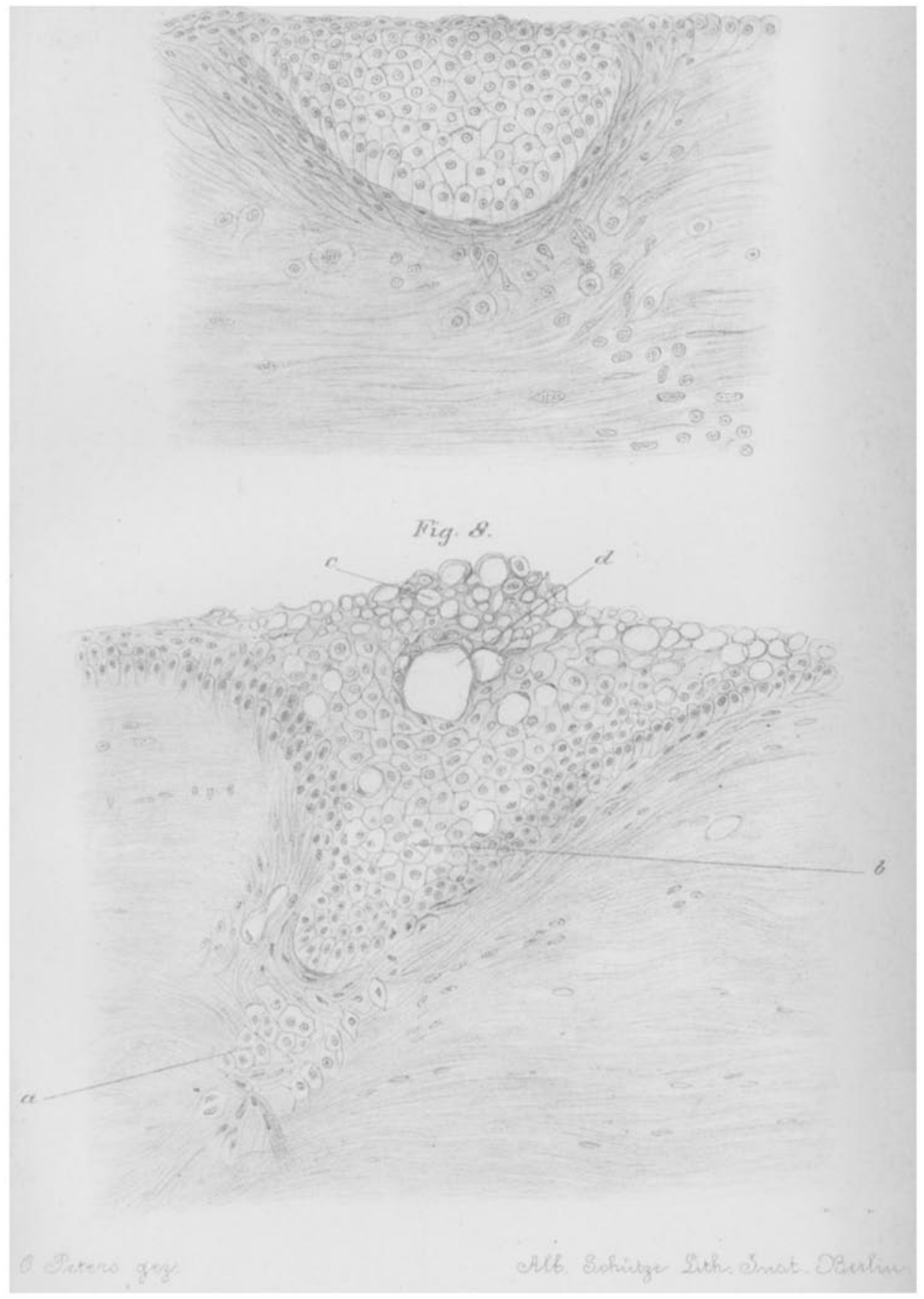

